\title{
NOTE INTORNO AL TEOREMA DI SHANNON
}

\section{Silvio De Francesco}

\section{Paragrafo $1^{\circ}$}

Le considerazioni che faremo di consentiranno di mettere in evidenza i fondamenti del Teorema Shannon, le condizioni per la sua validita, la natura della convergenza della formula di Shamon, l'errope ad essa relativo.

Von i superfluo arvertire che essendo le seguenti note redatte da un tecnico delle Telecomunicazioni, esse non hanno quel linguaggio ortodos:o che si richiede dai matematici puri: perciò l'autore chiede fin da questo momento venia e comprensione. Avvertiamo inoltre che verra spesso usato il simbolo $F\}(f(t)\}$ per esprimere la trasformata di Fourier della funzione del tempo entro parentesi a graffa funzione di variahile reale), nonché il simbolo $F^{1}\{F(\omega)\{$ per indicare la traformata inversa o antitrasformata di Fourier della funzione $F($ (s) $)$ di (1), in cui $F(a)$ i la trasformata di Fourier della funzione f(t).

\section{Paragrafo $2^{\circ}$}

Riassumiamo brevemente la dimostrazione del teorema in discoro, data dallo Shannon stesso nel: Proceedings of the IRE - Communications in the presence of noise - gennaio 1949, volume 37, n. 1, paw. 10. is suntergriato ciò che interessa le presenti note.

Ecro l'enunciato originario del teorema: se una funzione $f(t)$ non conticne frequenze più alte di w, essa è completamente determinata -e si conoscono i valori che essa assume in istanti spaziati fra loro di $\frac{1}{2 w}(1)$.

11) Ciò vale quanto asserire che se una funzione della variabile $t, f(t)$, è tale the la sua trasormata di Fourier $F(11)$ risulti limitata allintervallo d'esistenza $(-2-\pi, 2 \pi w)$, dove $(1)=2-n$, la funzione $\dot{e}$ completamente determinata se si ronoscono i valori che essa prende in punti spaziati tra loro di $\frac{1}{2 w}$. 
Partendo dalle formule:

$$
f(t)=\int_{-\infty}^{\infty} F(\omega) e^{\mathrm{j} \omega t} d \omega, F(\omega)=\frac{1}{2 \pi} \int_{-\infty}^{\infty} f(t) e^{-\mathrm{j} \omega t} d t
$$

si supponga che la trasformata di Fourier di $f(t)$, ossia la $F\left(\omega^{\prime}\right)$ sia limitata all'intervallo $(-2 \pi w, 2 \pi w)$.

Si possono allora sostituire ai limiti del primo integrale dell: [1] le pulsazioni $-2 \pi w, 2 \pi w$ : si effettui inoltre la sostituzione $t=\frac{n}{2 w}$ (n intero) ottenendo cosi la seguente espressione:

$$
f\left(\frac{n}{\Sigma w}\right)=\int_{-2 \pi w}^{2 \pi w} F(\omega) e^{\mathrm{j} \frac{\mathrm{n} \omega}{2 w}} d \omega
$$

Si osservi che questa relazione ha il $2^{\circ}$ membro uguale, a meno del fattore $1 / 4 \pi w$ al coefficiente ennesimo dello sviluppo in serie di Fourier della:

$$
F(\omega)=\Sigma_{n} C_{n} e^{j \frac{n \omega}{2 w}}, \text { ossia } c_{n}=\frac{1}{4 \pi \omega} \int_{2 \pi v}^{2 \pi \omega} F(\omega) e^{\mathrm{j} \frac{\mathrm{n} \omega}{2 w}} d \omega
$$

Possiamo dunque scrivere:

$$
J\left(\frac{n}{2 w}\right)=\int_{-2 \pi \omega}^{2 \pi w} F(\omega) e^{3 \frac{\mathrm{n} \omega}{2 w}} d \omega=4 \pi w C_{n}=x_{n}
$$

ciò̀ $x_{n} \grave{e}$ ugúule al valore di $f(t)$ nell'istante $\frac{n}{2 w}$.

Una volta conosciuti i coefficienti della serie di Fourier della $F((1))$, questa resta determinata e con essa la $f(t)$. Ma poiché detti coefficienti sono eguali ai valori che $f(t)$ assume nell'istante generico $\frac{n}{2 w}$. la $f(t)$ è completamente determinata qualora si conoscano i valori che essa prende in istanti spaziati tra loro di $\frac{1}{2 w}$. Essa può essere ricostruita per mezzo di questi valori usando funzioni del tipo $\frac{\sin 2 \pi w t}{2 \pi w t}$ 
di spetto costante nella handa we nullo all'infuori $\left.{ }^{2}{ }^{2}\right)$. Infatti una funzione del tipo:

$$
\sum_{-\infty} x_{n} \frac{\sin \pi(2 w t-n)}{\pi(2 w t-n)}
$$

ha la proprieta d'assumere valori uguali ad $\int\left(\frac{n}{(2 w}\right)$ negli istanti $t=\frac{n}{2 w}$ ed ha la stessa ampiezza di spettro (ossia intervallo di esistenza) di fill.

Di tali funzioni ve n’è una ed una sola ("). Nel caso che la funzione $f(t)$ sia nulla all'esterno dell'intervallo $(o, T)$ cioè se essa rappresenta un segnale di durata limitata si avrà:

$$
f(t)=\sum_{1}^{2 w \mathrm{~T}} x_{n} \frac{\sin \pi(2 w t-n)}{\pi(2 w t-n)} .
$$

\section{Paragrafo $3^{\circ}$}

Ricerehiamo le condizioni necessarie e sufficienti affinche la formula di Shannon s'identifichi con uno sviluppo in serie di funzioni ortoronali $\left(^{\text {th }}\right.$ converente completamente in m. (I. (media quadratica) verso f(l). Tale assunto potrel,be sembrare arlitrario; ma se si pensa che sono gli sviluppi ortogonali quelli che assicurano la migliore appossinazione in m. I. (e che si tratti di ciò risulta chiaramente dal citato articolo di Shamnon) tale ricerca appare pienamente griustificata.

Per semplificare la serittura poniamo

$f\left(\frac{n}{2 w}\right)=f_{n}, \frac{\sin \pi(2 w 1-n)}{\pi(2 w t-n)}=\frac{\sin 2 \pi w\left(t-\frac{n}{2 w}\right)}{2 \pi w\left(1-\frac{n}{2}\right)}=\sigma_{n}, \quad \Sigma_{n}$ per $\Sigma_{n}$ Isalvo diversamente specificato)

(2) Ossia la trasformata di Fourier della funzione $\frac{\sin 2 \pi w t}{2 \pi u t}$, e una lunzione ad andamento rettangolare. limitua allintervallo desistenza $(-2-\pi \cdot 2 \pi x)$.

(3) Nel senso: il rui spettro sia limitato alla banda w e rhe passi per i valori

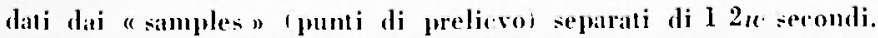

(t) Questa idea fu sugzerita allautore delle presenti note dal Comandante Riccardo Bignamini per mettere in lue la ragione dellintervallo $r a$ di preliexo dei valori di $f(1$. Ringrazio il Bignamini rhe spero voglia portare a termine un inte. ressante sudio sul significalo numerico del Teorema di Shannon e connessi. 
e, conservando le ipotesi di cui al par. precedente, cominciamo con losservare, insieme allo Shannon, che il sistema $/ / \sqrt{2 w} \sigma_{n}$ i ortonormale in $(-\infty, x)$. Lo sviluppo in discorso, che prende allora la forma $\Sigma_{n} f_{n} \sigma_{n}$ e che può anche scriversi $\sum_{-\frac{f_{n}}{1 / 2 w}}\left(1 / \overline{2 w} \sigma_{n}\right)$, sarà orto. gonale se $\frac{f_{n}}{\sqrt{2 w}}=\int f(t) \sqrt{2 w} \sigma_{\mathrm{n}} d t$, ossia se

$$
f_{\mathrm{n}}=2 w \int_{-\infty}^{\infty} f(t) \sigma_{\mathrm{n}} d t=\int_{-\infty}^{\infty} f(t) \frac{\sin 2 \pi w\left(t-\frac{n}{2 w}\right)}{\pi\left(t-\frac{n}{2 w}\right)} d t
$$

Riservandoci di dimostrare, al prossimo paragrafo, come nell'ipotesi del Teorema (e con altre precisazioni) l'ultima condizione risulti effettivamente verificata, notiamo che, se $f(1)$ i a quadrato sommabile in $(-\infty, \infty)$ vale la limitazione di Bessel

$$
\Sigma_{\mathrm{n}}\left(\frac{f_{\mathrm{n}}}{\sqrt{2 w}}\right)^{2} \leq \int_{-\infty}^{\infty} t^{2}(t) a t
$$

Come puó dedursi dal contesto della dimostrazione data da E. W. Holson a proposito del Teorema di Parseval esteso ad un intervallo infinito; infatti non essendo provato che il sistema $\sqrt{2 w} \sigma_{n}$ sia completo nel tratto $(-\infty, \infty)$ non varà in genere il segno di uguaglianza. Per il citato Teorema, efr. E. W. Hohson. The Theory of functions of a real variable. Vol. II, 2: ed. 1926 par 402, parr. 759. 60-61 (i).

Dopodiché, visto che il sistema $/ / \sqrt{2 w} \sigma_{n} ! \dot{i}$ ortonommale in $(-\infty, \infty)$ la serie $\Sigma_{n} f_{n} \sigma_{n}$ convergera in m. q. verso una funzione $f(t)$ a quadrato sommabile in $(-x, \infty)$ che ha por coefficienti di Fourier gli $\frac{f .}{\sqrt{2 u}}$ ossia $\frac{f_{\mathrm{n}}}{\sqrt{2 u}}=\int f(\bar{t}) \sqrt{2 u} \sigma_{\mathrm{n}}$ at (per il Teorema Riesz-Fischer esteso ad intervallo infinito: efr. il ritato Hohson pay. 761, 2 par 493 i.

(5) Cfr. anche G. Vital. Geometrin nello spazio hilbertimo. 
Poiché però non ì dimostrato che il sistema $\mid \sqrt{2 w} \sigma_{n} \nmid$ è completo to chiusol in $(-\infty, x)$, non puo dirsi che $f(t)$ sia unica (cfr. il cilato Hohson pag. 762 ).

Si noti tuttavia che se $\int_{-\infty}^{\infty}|f(t)| d t$ i convergente (i), allora ad $F(0)$, quale trasformata di Fourier di $f(t)$, corrisponde unicamente la $f(t)$; se poi vale l'ipotesi che la $F ; f(t)\}=F(\omega)$ sia limitata all'intervallo d'esistenza finito $1-2 \pi w, 2 \pi w)$, allora a causa di cio e della supposta assoluta integrabiliti di $f(t)$ in $(-x, 2$ vale la relazione

$$
f(t)=\sum_{-\infty}^{\infty} f(\tau) \frac{\sin 2 \pi \omega(t-\tau)}{\pi(t-\cdots)} d \tau
$$

refr. par. successivo per la dimostrazionel.

Ora il secondo membro di $[1]$ i la $F^{-1}$ di $R(\omega ; 22 \pi u) \cdot F(\omega)$; in ('ui

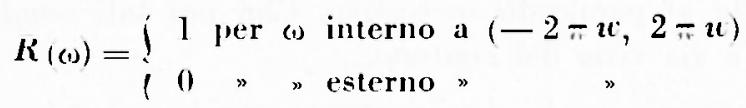

ed $F^{-1} ; R((1)) ;=\frac{\sin 2 \pi \pi t}{l}$ : segue di qui, per l'unicità di $f(t)$ rispetto ad $F((1)$, che la [1] vale soltanto se solto il segno d'integrale appare la $f$ e non unaltra funzione come ad esempio $\bar{f}$. Ma gli $f_{\mathrm{n}}$ si ollengono dalla $[1]$ ponendo $t=\frac{n}{2 a}$ : infalti

$f\left(\frac{n}{2 w}\right)=\int_{-\infty}^{\infty} j(\tau) \frac{\sin 2 \pi w\left(\frac{n}{2 w}-\tau\right)}{\pi\left(\frac{n}{2 w}-\tau\right)} d \tau=\int_{-\infty}^{\infty} j(\tau) \frac{\sin 2 \pi w\left(--\frac{n}{2 w}\right)}{\pi\left(\tau-\frac{n}{2 w}\right)} d \tau$

(t) Overo a variazione limitata purchè $l m f f(t)=0$; naturalmente $f(n)$ deve esere finiti e regolare per 1 finito ed anche sommabile in qualunque tratto finito. Cfr. G. VItald e G. Sinsont, Moderna teoria delle funzioni di variabile reale. 3:1 ediz. 1952, Parte II, pag. 163.1. 
e cambiando $\tau$ in $t$.

$$
f\left(\frac{n}{2 w}\right)=\int_{-\infty}^{\infty} f(t) \frac{\sin 2 \pi w\left(t-\frac{n}{2 w}\right)}{\pi\left(t-\frac{n}{2 w}\right)} d t
$$

si conclude che $\bar{f}(t)=f(t)$ e cle quindi la ricercata convergenza in m. q. è completa.

Riassumendo, le condizioni necessarie al nostro assunto sono:

a) $f(t)$ a quadrato sommabile in $(-\infty, \infty)$

b) $F(w)$ linitata allintervallo finito d'esistenza $(-2 \pi w, 2 \pi w)$

c) f(t) assolutamente integrabile in $1-2,2$, o equivilente (clir. nota precedente).

Alla bl può sostituirsi la

$$
\left.b^{\prime}\right) f(t)=\int_{-\infty} f(\tau) \frac{\sin 2 \pi(t-\tau)}{\pi(t-\tau)} d \tau \text {, con le considerazioni che }
$$

saranno fatte al paragrafo successivo. Che poi tali condizioni siano sufficienti s̀è gia visto dal contesto.

\section{Paragrafo $4^{\circ}$}

Dimostriamo ora quanto preannunciato nel par. 3.

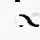

Se $f(t)$ e tale che $\int|f(t)|$.dt $\tau^{\tau}$, converga e che $F: f(t) !=F(\omega)$

risulti limitata allintervallo $(-2 \pi w, 2 \pi w)$ con $w$ finito e positivo $1^{\infty}$, allora vale la relazione:

$$
f(t)=\int_{-\infty}^{\infty} f(\tau) \frac{\sin 2 \pi \omega(t-\tau)}{\pi(t-\tau)} d \tau
$$

(i) Vedi note al par. precedente.

(8) w rappresenta la frequenza delle oscillazioni sinusoidali in cui puo soum. porsi la $f(t)$, perciò la condizione $w>0$ non è una limitazione, ma ¿ prifellat. mente aderente alla realta fisica, in cui non si concepiscono frequenze negative. 
Dalle formule

$$
f(t)=\int_{-2 \pi \omega}^{2 \pi \omega} F(\omega) e^{j \omega t} d \omega, \quad F(\omega)=\frac{1}{2 \pi} \int_{-\infty}^{\infty} f(t) e^{-\mathrm{j} \omega t} d t
$$

con metodo di iterazione si ottiene lequazione tche formalmente i un'erpuzione integrale)

$$
f(t)=\int_{-2 \pi u}^{2 \pi u} \epsilon^{\mathrm{j} \omega \mathrm{t}}\left[\frac{1}{2 \pi} \int_{-\alpha}^{\alpha} f(t) e^{-\mathrm{j} \omega \mathrm{t}} d t\right] d \omega .
$$

Osservando che:

$\left.\int_{-2 \pi \omega}^{2 \pi t \omega} e^{j \omega t} \mid \frac{2}{2 \pi} \int_{-\infty}^{\infty} f(t) e^{-j \omega t} d t\right] d(1)=\int_{-\infty}^{\infty} R(\omega) e^{j \omega t}\left[\frac{1}{2 \pi} \int_{-\infty}^{\infty} f(t) e^{-j \omega t} d t\right] d \omega$

se

$$
R(\omega)= \begin{cases}1 \text { per } \omega \text { interno a }(-2 \pi w, 2 \pi w) \\ 0, \text { esterno }\end{cases}
$$

la [1] potrà porsi cguale a

$\left.\int_{-\infty}^{\infty} R(\omega)\left[\frac{1}{2 \pi} \int_{-\infty}^{\lambda} f(t) e^{j \omega t} d t||_{\mid}^{j(\omega t} d(1)=-F-1|R(\omega)| \frac{1}{-\pi} \int_{-\infty} f(t) e^{-j \omega t} d t\right]\right\}$

Ma

$$
R((1))\left[\frac{1}{2 \pi} \int_{-\infty}^{\infty} f(t) e^{-\mathrm{j} \omega t} d t \mid=F\left\{\frac{1}{2 \pi} \int_{-\infty}^{\infty} \frac{2 \sin 2 \pi w(t-\tau)}{t-\tau} f(\tau) d \tau\right\}\right.
$$

iper il teorema del prodotto integralei essendo 
l'antitrasformata di $R(\omega)$ cosicché:

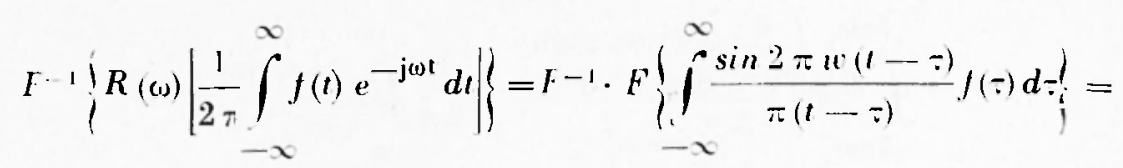

$$
\begin{aligned}
& =\int_{-\infty}^{\infty} f(\tau) \cdot \frac{\sin 2 \pi u(\cdots)}{\pi(t-\tau)} d \tau
\end{aligned}
$$

che i: quanto ei proponevamo di dimostrare.

Osservando il metodo tenuto nel dimostrare la [1], si vede che. essendo $F(\omega)$ limitata all'intervallo d'esistenza $(--2 \pi w, 2 \pi w)$ (e nulla al di fuori), varrà pure la relazione

$$
\int_{-2 \pi \omega}^{2 \pi \omega} F(\omega) e^{j \omega t} d \omega=\int_{-\infty}^{\infty} R^{\prime}(\omega) F(\omega) e^{j \omega^{\prime}} d \omega
$$

dove

$$
R^{\prime}(\omega)=\left\{\begin{array}{l}
1 \text { per } \omega \text { interna } a\left(-2 \pi w^{\prime} ; 2 \pi w^{\prime}\right) \\
0 \% \text { esterno } "
\end{array}\right.
$$

con $w^{\prime}>w$, in quanto compare sempre sollo il segno di integrale la funzione $F(\omega)$.

Ciò porterehbe a conchudere che la $f(t)$, nelle ipotesi del teorema. è sviluppalile in serie convergente mediante ogni sistema ortonormale

$$
\left\{\sqrt{2 w^{\prime}} \frac{\sin \pi\left(2 w^{\prime} t-n\right)}{\pi\left(: w^{\prime} t-n\right)}\right\}
$$

purché $w^{\prime}=$ : 11 sistema caratterizzato da $w^{\prime}=w$ ì però quello che permette il prelievo meno frequente di valori della $f(t)$, ma per converso è quello che offre coincidenze meno frequenti tra lo sviluppo e la funzione sviluppata (cfr. nota (') al par. 2).

\section{Paragrafo $5^{\circ}$}

Partendo dalla formula $f(t)=\int_{-\infty}^{\infty} f(\tau) \frac{\sin 2 \pi w(t-\tau)}{\pi(t-\tau)} d \tau$ e con le stesse ipotesi di cui al paragrafo $4^{\prime \prime}$ si puo mostrare come la formula 
di Shannon isviluppo di Shannon uguagli effettivamente una funzione p(t) tale che:

$$
2 w \int_{o}^{12 w} \varphi(\tau-t) d \tau=f(t)
$$

Infatti

$$
f(t)=\int_{-\infty}^{\infty} f(\tau) \frac{\sin 2 \pi w(t-\tau)}{\pi(t-\tau)} d \tau=\Sigma_{\mathrm{n}} \int_{\Gamma \lambda}^{(\mathrm{a}+1) \lambda} \frac{\sin 2 \pi w(t-\tau)}{\pi(t-\tau)} j(\tau) d \tau
$$

con $n$ intero, $\lambda$ finito e costante; ma

$$
\int_{n \lambda}^{(n-1) \lambda} \frac{\sin 2 \pi w(t-\tau)}{\pi(t-\tau)} f(\tau) d \tau=\int_{0}^{\lambda} \frac{\sin 2 \pi w(t-\tau-n \lambda)}{\pi(t-\tau-n \lambda)} f(\tau+n \lambda) d \tau
$$

per cui

$$
f(l)=\Sigma_{n} \int_{0}^{\lambda} \frac{\sin 2 \pi w(t-\tau-n \lambda)}{\pi(t-\tau-n \lambda)} j i+\pi \lambda j \dot{u} \tau
$$

Usserviamo che per $\lambda=1 / 2 u$, la $\because \frac{\sin 2 \tau u(t-\tau n \lambda)}{\tau(t-\tau-n \lambda)} j(\tau-n \lambda)$ per il teorema di Shannon, e certamente convergente in m. q. completamente verso $2 \pi f(t-\tau)$ e quindi, ammessa la permutabilita degli operatori L e nel nostro caso, cioè che $L . J=\int \cdot$ Spotremo serivere

$$
\begin{aligned}
& \sum_{0}^{\lambda} \frac{\sin 2 \pi w(t-\tau-n \lambda)}{\pi(t-\tau-n \lambda)} f(\tau-n) d \tau= \\
= & \int_{0}^{\lambda}\left[\Sigma_{n} \frac{\sin 2 \pi w(t-\tau-n \lambda)}{\pi(t-\tau-n \lambda)} f(\tau-n \lambda)\right] d \tau
\end{aligned}
$$

e quindi

$$
f(t) \simeq \frac{1}{\lambda} \int_{0}^{\lambda} f(t-\tau) d \tau
$$


Appare ovvio che quest'ultima è una formulazione asintotica della nota formula del valor medio.

E chiaro che la sommatoria in discorso eguaglia allora l'altra funzione $\frac{1}{\lambda} \varphi(\tau-t)$ tale che

$$
\frac{1}{\lambda} \int_{0}^{\lambda} \varphi(t-\tau) i \tau=f(1) \operatorname{con} \lambda=1 / 2 \omega
$$

da ciò possiamo dedurre che lo sviluppo di Shamnon eguaglia una funzione $p(t)$ tale che

$$
2 w \int_{\varphi(t-\tau) d \tau=f(t)}^{1 / 2 u}
$$

\section{Paragrafo $6^{o}$}

Cerchiamo ora d'invertire l'integrale $\frac{1}{\lambda} \int_{0}^{0} \varphi(t-\tau) d \tau=f(t)$ dove con $\lambda$ si indica $1 / 2 u$.

Con un cambiamento di origine, si può passare alla:

$$
f(t)=\frac{1}{\lambda} \int_{-\frac{\lambda}{2}}^{\frac{\lambda}{2}} \varphi\left(t-\tau+\frac{\lambda}{2}\right) d \tau
$$

e ponendo $\lambda=2 \alpha$ si ottiene:

$$
f(t)=\frac{1}{2 \alpha} \int_{-\infty}^{\alpha} \varphi(t-\tau+\alpha) d \tau=\frac{1}{2 \alpha_{t}} \int_{0}^{ \pm \alpha} \varphi(t-\tau) d \tau
$$

Da quest'ultime cercheremo di operare l'inversione dello integrale per due vie:

$$
\text { 1) } f(t)=\frac{1}{2 \alpha} \sum_{-\alpha} r(\tau ;-\alpha, \alpha) \varphi(1-\tau+\alpha) d \tau
$$


love

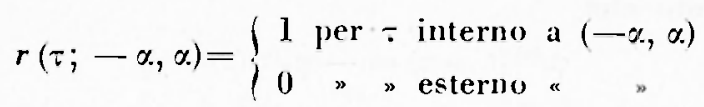

e quindi $F: f(t):=\frac{1}{2 \alpha} \quad \frac{2 \sin \omega \alpha}{(1)} \Phi(\omega) e^{j \omega \alpha}=\frac{\sin \omega \alpha}{\omega \alpha} \Phi(\omega) e^{j \omega \alpha}$, dove. $\Phi(\omega)=F ; \varphi(t) ;(\%)$ Indicando con $F(\omega)$ la $F: f(t)\}$ si ottiene:

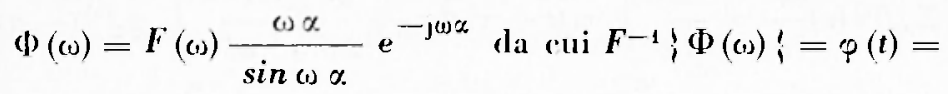

$$
\begin{aligned}
& =\frac{1}{2 \pi} \int_{-2 \pi w}^{2 \pi v} F(\omega) \frac{\omega \alpha}{\sin \omega \alpha} e^{-j \omega \alpha} \epsilon^{j(\omega)} d \omega
\end{aligned}
$$

a causa della natura $\mathrm{F}(\omega)$ (limitata cioe all'intervallo $-2 \pi w, 2 \pi w$ ) - ricordando che $2 \alpha=\frac{1}{2 w}$ si ha in definitiva :

$$
\varphi(t)=\frac{1}{2 \pi} \int_{-\pi / 2 \alpha}^{\pi 2 \alpha} F(\omega) \frac{\omega \alpha}{\sin \omega \alpha} e^{-i \omega \alpha} e^{j \omega t} d \omega
$$

Notando che $\frac{\omega \alpha}{\sin \omega \alpha}$ \& finita nell'intervallo $(-\pi / 2 \alpha . \pi / 2 \alpha)$ si conclude che l'integrale a secondo membro della [1] ¿ proprio.

$$
\text { 1) } \begin{gathered}
f(t)=\frac{1}{2 \alpha} \int_{0}^{2 \alpha} \rho(t-\tau) d \tau \\
f_{\mathrm{t}}^{\prime}(t)=\frac{1}{2 \alpha} \int_{\alpha}^{2 \alpha} \varphi_{\mathrm{t}}(t-\tau) d \tau \\
\vdots \\
f_{\mathrm{t}}^{(\mathrm{t})}(\mathrm{l})=\left.\frac{1}{2 \alpha}\right|_{0} ^{2 \alpha} \varphi_{1}^{(\mathrm{nt})}(t-\tau) d \tau .
\end{gathered}
$$

(9) In questa analisi si sono adottate le formule generiche di trasformazione

$$
g(t)=\frac{1}{2} \pi \int_{-\infty}^{\infty} G(\omega) e^{j \omega t} d \omega ; G(\omega)=\int_{-\infty}^{\infty} g(t) e^{-j \omega t} d t,
$$

come si usa spesso nelle trattazioni sui filtri elettrici. 
Ma fintanto che

$$
\varphi_{1}^{(n)}(t-\tau)=-\varphi_{\tau}^{(n)}(t-\tau)
$$

e quindi ammesso che $f(t)$ sia sviluppabile in serie di potenze intere nell'intorno di $t=o$, si ha

$f(t)=\Sigma_{\mathrm{D}} f^{(\mathrm{v})}(\mathrm{o}) \frac{t^{\mathrm{n}}}{n !}=\frac{1}{2 \alpha} \int_{0}^{2 \alpha} \varphi(t-\tau) d \tau-\frac{1}{2 \alpha} \sum_{\mathrm{n}}^{\infty} \frac{t^{\mathrm{n}}}{n !} \int_{j^{\prime}}^{2 \alpha} \varphi_{\tau}^{(n)}(-\tau) d \tau$

Tenendo ora conto che

$$
\int_{0}^{2, \imath} \varphi^{(n)}(-\tau) d \tau=\left[\varphi^{(n-1)}(-\tau)\right]_{0}^{2 \alpha}
$$

avremo

$f(t)=\frac{1}{2 \alpha} \int_{0}^{2 \alpha} \varphi(-\tau) d \tau-\frac{1}{2 \alpha}\left\{[\varphi(-\tau)]_{\alpha}^{2 \alpha} \cdot t+\ldots+\left[\varphi^{(n-1)}(-\tau)\right]_{\alpha}^{2 \alpha} \frac{t^{\mathrm{n}}}{n !}+\ldots\right\}$

e derivando $f(t)$ rispetto a $t$ otterremo:

$$
\begin{aligned}
f^{\prime}(t) & =\frac{1}{2 \alpha}\left\{\varphi(2 \alpha)+\ldots+\varphi^{(n-1)}(2 \alpha) \frac{t^{n-1}}{(n-1) !}+\ldots\right\}+ \\
& \left.-\frac{1}{2 \alpha} ! m(o)+\ldots+\varphi^{(n-1)}(o) \frac{t^{n-1}}{(n-1) !}+\ldots\right\}
\end{aligned}
$$

Ossia, ammesso che $\varphi(t)$ sia sviluppabile in serie di potenze intere negli intorni di 0 e di $2 \alpha$, risulterebbe

$$
f^{\prime}(t)=\frac{1}{2 \alpha} \varphi(t+2 \alpha)-\frac{1}{2 \alpha} \varphi(t)
$$

Prendendo la trasformata di Fourier di quest"ultima relazione si ottiene:

$$
\begin{gathered}
F\left\{f^{\prime}(t)\right\}=j \omega F(\omega)=\Phi(\omega) \frac{e^{2 \mathrm{j} \omega \alpha}}{2 \alpha}-\Phi(\omega) \frac{1}{2 \alpha}=\bar{\omega}\left(\omega, \frac{e^{\mathrm{j} \omega \alpha}-e^{-\mathrm{j} \omega \alpha}}{2 \alpha} \cdot c^{\mathrm{j} \omega \alpha}\right. \\
\operatorname{con} \Phi(\omega)=F \nmid \varphi(t)\}, \text { da cui : } \Phi(\omega)=F(\omega) \frac{\omega \alpha}{\sin \omega \alpha} e^{-\mathrm{j} \omega \alpha}
\end{gathered}
$$


e quindi

$$
p(t)=\frac{1}{2 \pi} \int_{-\pi / 2 \alpha}^{\pi / 2 \alpha} F(\omega) \frac{\omega \alpha}{\sin \omega \alpha} e^{-j \omega \alpha} e^{j \omega t} d \omega
$$

Ammesso dunque che $f(t)$ sia tale da permettere tutti i passaggi fin qui usati, saremo ora in grado di dare una espressione formale dellerrore $=$ inerente all'uso della formula di Shannon e cioè:

$$
\begin{gathered}
\equiv=f(t)-p(t)=f(t)-\frac{1}{2 \pi} \int_{-\pi 2 \alpha}^{\pi 2 \alpha} F(\omega) \frac{\omega \alpha}{\sin \omega \alpha} e^{-j \omega \alpha} e^{j \omega t} d \omega= \\
=\frac{1}{2 \pi} \int_{-\pi 2 \alpha}^{\pi 2 \alpha} F(\omega)\left(1-\frac{\omega \alpha e^{-j \omega x}}{\sin \omega \sigma}\right) e^{i \alpha t} d \omega
\end{gathered}
$$

ovvero, se ci riferiamo alle notazioni usate nel teorema di Shannon:

$$
\Sigma=\frac{1}{2 \pi} \int_{-\pi w}^{2 \pi w} F(\omega)\left(1-\frac{\omega / 4 w e^{-\mathrm{i} \omega / 4 w}}{\operatorname{in} \omega / 4}\right) e^{\mathrm{i} \omega \mathrm{t}} d \omega .
$$

\section{RIASSUNTO}

Fengono messi in evidenza i fondamenti del teorema di Shannon, dopo aver stabilito una proprieta relativa alla trasformata di Fourier di funzioni il cui spettro risulti limitato. Viene pure ricercata una espressione formale dellerrore inerente allo sviluppo, limitata però dalla validità dei passaggi usuti. Tanto la proprietà esposta al par. 4, quanto la dimostrazione del teorema di Shannon data al par. 3 a le successive elaborazioni risulterebbero nuove.

\section{SUMMARY}

The well-knoun formula of this theorem is demonstrated as a case of complete convergency of a generalized Fourier's expansion, with the aid of a theorem which is proved in section 4. An expression of the inherent error is given in section 6, provided that the hypothesis formulated therein are accomplished. The arguments of sections 3,4 etc. (so far as the author is anare) seem to have not been treated till notv. 